\title{
La industria cultural en las ciudades de México: Los 'servicios simbólicos intensivos en conocimiento' (sIC-simbólicos)
}

\author{
Boris Graizbord. El Colegio de México, Ciudad de México, México. \\ Luis-Enrique Santiago. Universidad Autónoma de Aguascalientes, Aguascalientes, \\ México.
}

RESUMEN | En este trabajo reflexionamos sobre el potencial de la "industria cultural" para dinamizar la economía de las ciudades en México. Las actividades de orden cultural han sido promovidas en grandes ciudades para revitalizar sus economías y, en algunos casos, aportan un elevado porcentaje de su empleo e ingreso. Representan, además, un nicho que atrae visitantes y genera utilidades, y se han convertido en su marca y sello simbólico. El objetivo central es estudiar la participación y los cambios recientes de las actividades culturales en la estructura económica de las 384 ciudades del Sistema Urbano Nacional (sun) mexicano. Metodológicamente, tales actividades han sido definidas como 'servicios intensivos en conocimiento' (knowledge-intensive services, KIs) que producen elementos 'simbólicos': medios de comunicación masiva, cultura y diseño. Los resultados indican que no solo las grandes ciudades tienen concentraciones sobresalientes de KIs-simbólicos, sino también las medianas y pequeñas, lo cual abre un abanico de posibilidades para implementar políticas públicas encaminadas a su desarrollo en el ámbito urbano.

PALABRAS ClaVe | sistema urbano, sociedad del conocimiento, política urbana.

ABSTRACT | In this paper we reflect on the potential of the "cultural industry" to boost the economy of Mexican cities. Cultural activities have been promoted in large cities to revitalize their economies. In some cases, cultural activities contribute to the local economy with high percentage of their employment and income. These activities also represent a niche that attracts visitors, leave profits, and have become its symbolic brand. The main objective is to study the participation and changes of cultural activities in the economic structure of the 384 cities of the National Urban System (NUS) between 2004 and 2014. Methodologically, such activities have been defined as 'knowledge-intensive services' (KIS) that produce 'symbolic' elements: mass media, culture and design. The results indicate that not only large cities have significant KIS-symbolic concentration; medium-sized and small ones do as well. This result opens possibilities to implement public policies that focus on the development of KIS-symbolic in the local economy.

KEYWORDs | urban system, knowledge society, urban policy. 


\section{Introducción}

Las ciudades -especialmente las más grandes- han adquirido un estatus protagónico no solo porque serán receptoras del crecimiento esperado de la población mundial en los próximos años (Graizbord, 2007), sino porque se ven como solución para los problemas sociales, económicos y ambientales que se enfrentan en esta "era urbana" (Burdett \& Philipp, 2018; Glaeser, 2012). Pero, además, porque en ellas se concentra la "clase creativa", que incluye no solo científicos, tecnólogos o empresarios, así como profesionales en educación y salud, sino también trabajadores en actividades culturales, creativas y de entretenimiento (Currid \& Connolly, 2008; Florida \& Mellander, 2014, p. 1). La importancia de este último grupo de actividades radica en su aportación económica, su capacidad de transformar el espacio urbano y su impacto significativo en el campo de las actividades económicas, sociales y urbanas (Amin \& Thrift, 2007; Florida, 2005; Hall, 2000; Scott, 2014; Storper \& Scott, 2009). El caso paradigmático es Londres, donde las actividades culturales y relacionadas con el ocio representan una proporción muy considerable de la actividad generadora de sus ingresos. En 2015 , Londres concentraba $47,4 \%$ ( $£ 42,0$ billones) del valor agregado bruto generado por las industrias creativas del Reino Unido, las cuales representaron directamente $11,1 \%$ de su economía local, con un ritmo de crecimiento superior al promedio nacional (Rocks, 2017, p. 46). Pero existen otras ciudades donde dichas actividades han adquirido un papel central en sus economías urbanas; por ejemplo, Bilbao, Barcelona, Glasgow, Los Ángeles, Nueva York y Buenos Aires, como se expone más adelante.

En este trabajo, las actividades culturales, creativas y de entretenimiento son enmarcadas como parte del sector servicios, especialmente como 'servicios intensivos (o de alto contenido) en conocimiento'1 (Graizbord \& Santiago, 2019). De acuerdo con Martin y Moodysson (2011, p. 1189), las industrias creativas/culturales son aquellas cuyo principal fundamento es el conocimiento simbólico. Se trata del conocimiento asociado a los atributos estéticos de los productos, la creación de diseño e imágenes y el uso económico de varias formas de artefactos culturales. Este tipo de conocimiento es incorporado y transmitido a través de símbolos estéticos, imágenes, signos, artefactos, sonidos y narrativas (Asheim, 2007, p. 226). Los cambios tecnológicos que recientemente han ocurrido -y continuarán desarrollándose- definen este grupo de servicios como parte de las 'industrias del futuro' (Avent, 2016; Ross, 2017). Consideramos la ciudad como el ámbito o milieu donde es posible ampliar, desarrollar e intercambiar el conocimiento, la creatividad y, por tanto, la innovación. Y el desarrollo de la cultura encuentra en la ciudad el medio propicio (Camagni, 2016; 2017).

1 Los 'servicios intensivos en conocimiento' (KIS, por sus siglas en inglés: knowledge-intensive services) son definidos como industrias de servicios en las que se llevan a cabo complejas operaciones de naturaleza intelectual desarrolladas por los individuos ocupados en ellas cuya principal característica es contar con altos niveles de calificación o expertise profesional, relacionado con un dominio técnico y funcional específico (Graizbord \& Santiago, 2019; Shearmur \& Doloreux, 2008). Sobre los distintos 'servicios intensivos en conocimiento' y su función como actividades clave en la generación de conocimiento analítico (científico), sintético (técnico-profesional) y simbólico (artístico), puede verse Santiago (2016, pp. 179-188). 
El objetivo central de este trabajo es analizar la participación y los cambios recientes en la estructura de la economía cultural en las ciudades de México en 2004 y 2014. Para ello se estudian los 'servicios intensivos en conocimiento' de tipo simbólico (de aquí en adelante, sic-simbólicos), los cuales han sido divididos en tres grupos: i) medios de comunicación masiva, ii) servicios culturales, y iii) servicios de diseño, empleando una metodología que permite identificarlos dentro de la estructura económica de las ciudades a partir del Sistema de Clasificación de América del Norte (scian) (Santiago, 2016). El análisis empírico se desarrolla utilizando información de microdatos de los Censos Económicos de 2004 y 2014 de las 384 ciudades que conforman el sistema urbano de México; elaboramos, además, un análisis estadístico que facilita conocer el "potencial" de cada ciudad y las variables clave que influyen en el desarrollo de los sic-simbólicos.

Las siguientes reflexiones contribuyen a enfatizar el papel relativo que pueden desempeñar la "cultura" y la creatividad en la revitalización de las economías urbanas en el caso de México. El estudio nos acerca a los cambios que ocurren en países en desarrollo, como México, consecuencia de su transformación económica hacia el sector servicios (Rodrik, 2016). Los resultados muestran que no solo las grandes ciudades del país (Ciudad de México, Guadalajara y Monterrey) son los principales núcleos de la actividad de los sic-simbólicos. Se identifica, en cambio, una serie de ciudades con perfiles urbanos, sociales y económicos específicos donde los sicsimbólicos encuentran el ámbito propicio para contar con importantes concentraciones espaciales y elevadas tasas de crecimiento. Los resultados ponen en cuestión la idea de las grandes ciudades como los espacios preeminentes para el desarrollo de este tipo de actividades y amplían las posibilidades de desarrollo de una política pública que busque aprovechar las posibilidades que ofrecen los servicios simbólicos en distintas ciudades del Sistema Urbano Nacional (sun).

El trabajo se estructura en cuatro apartados, además de la presente introducción. En el primero, se destacan la importancia del 'conocimiento' y del 'milieu' urbano como piezas clave en el desarrollo de los sic-simbólicos, los cuales son -y serán aún más- determinantes en la posición de las ciudades en la economía global del siglo xxi. En el segundo, se exponen los alcances y elementos metodológicos (fuentes de información, herramientas y ámbito de estudio) utilizados para el análisis empírico. En el tercero, se describen puntualmente los principales resultados que responden a las preguntas de investigación que guían el desarrollo del trabajo. En el cuarto, a manera de reflexiones finales, se presentan algunos lineamientos de política urbana dirigidos al desarrollo de ciudades especializadas en la producción de conocimiento simbólico.

\section{Marco conceptual}

\section{Ciudad como ámbito (milieu) de conocimiento, creatividad e innovación}

La sociedad global del siglo xxi experimenta un cambio paradigmático: "del estado nación a la ciudad" (Taylor, 2014). Según Sasaki (2008, p. 77), el "siglo de la ciudad" ha comenzado. En este marco se han desarrollado diferentes iniciativas urbanas orientadas a aprovechar los cambios que ocurren en la economía. Entre 
ellas: 'ciudades inteligentes', orientadas a aprovechar el desarrollo tecnológico (Komninos, Pallot \& Schaffers, 2013; Townsend, 2013); 'ciudades del conocimiento', que buscan impulsar el desarrollo de actividades intensivas en innovación (Yigitcanlar, Metaxiotis \& Carrillo, 2012); o 'ciudades creativas', cuyo objetivo central es aprovechar la producción cultural, junto con otras actividades intensivas en conocimiento (Florida, 2010; Scott, 2006, p. 1).

Todas las anteriores iniciativas reconocen la importancia de la congregación de firmas, en paralelo con el desarrollo de las tecnologías de la información y la comunicación (TIC) y el transporte. Y si bien estas últimas han estrechado las distancias espaciotemporales, sobre todo en el caso de firmas en industrias orientadas a la innovación, la concentración espacial permite formar clústeres que facilitan la derrama de conocimiento (Rosenthal \& Strange, 2001), en los cuales proliferan empresas pequeñas y altamente competitivas (Cowan, David \& Foray, 2000; Desrochers, 2001). En efecto, la proximidad física es la característica esencial de la difusión del conocimiento, pues facilita su intercambio. El logro de la transmisión interpersonal y efectiva del conocimiento, sea codificado y/o tácito, y su combinación correspondiente (aprehensión de su contenido, alcance de iguales conclusiones tras esta última), se alcanza mediante la relación mutua entre transmisor y receptor. La importancia latente de este proceso, de este derrame de conocimiento (knowledge spillover), está detrás de las economías de aglomeración y de la importancia económica de las ciudades.

En el terreno empresarial, la existencia de ferias, jornadas, reuniones, organización de grupos de trabajo, la convivencia en dicho terreno o fuera del mismo, ofrecen espacios para alcanzar la comunicación mutua facilitadora de los ajustes necesarios entre las cogniciones de diversos sujetos, de modo que la comprensión de lo que encierra el "paquete" tiende a ser común. Desde la perspectiva de la innovación empresarial, señala López (2009, p. 13), el conocimiento formal o codificado y el tácito producen combinaciones que el autor denomina conocimiento locacionaltraslacional. El conocimiento, dice, adquiere su forma peculiar como resultado de la fusión/hibridación de ambos tipos de conocimiento, con códigos particulares resultado del lenguaje científico, técnico y artesanal preexistentes en determinado tiempo y lugar. Es locacional, dado que resulta posible identificar su presencia en un espacio geográfico dado; y es simultáneamente traslacional, ya que posibilita la fusión/hibridación de diversas piezas de conocimiento, bien sean de naturaleza tácita o codificada, presentes en un espacio y tiempo determinados.

\section{Cultura, cultura urbana e industria cultural}

En el plano internacional, un ejemplo de ciudad que ha promovido la cultura como su base económica es Glasgow, que no es precisamente una "gran capital". Se ha estimulado en ella la actividad artística y las actividades culturales, convirtiendo las bodegas y las instalaciones industriales abandonadas en espacios que acogen usos residenciales y servicios culturales. Por supuesto, grandes capitales como París, Londres, Nueva York, ahora Berlín, y desde siempre Madrid para los latinoamericanos, son destinos turísticos para consumir cultura. Pero también en Bilbao, Barcelona y Sídney se promueve explícitamente dicha oferta. 
El Índice Global de Ciudades (Hales, Peterson, Mendoza \& Gott, 2014), clasificatorio de grandes ciudades, incluye algunas de América Latina que muestran un perfil diferenciado, de acuerdo con los criterios utilizados, cuya combinación otorga su lugar en la lista. Entre ellas, quizá la de mayor historia y prestigio en nuestro subcontinente sea Buenos Aires. Aparece esta seguida de Sáo Paulo y de la Ciudad de México. Ambas destacan en la participación del capital humano y de la "experiencia cultural" frente a las otras capitales incluidas. De hecho, Buenos Aires se sitúa en el octavo y el decimoprimer sitio en experiencia cultural y en capital humano, respectivamente, si bien en la lista de las sesenta que se incluyen en el Índice Global se ubica en el vigésimo lugar, mientras que las otras dos ciudades señaladas ocupan los lugares trigésimo cuarto y trigésimo quinto

Fomentar la cultura y la industria cultural como estrategias de desarrollo urbano, en los casos en que ha tenido éxito, se ha debido probablemente al crecimiento y proliferación de servicios que apoyan la economía en los medios, y al interés por parte de los organismos multilaterales (Unesco, entre otros), por proteger, cuidar y recuperar el patrimonio cultural, histórico-arquitectónico, e incluso intangible, y su enorme valor simbólico.

Lo anterior se da en el contexto de cuatro premisas (Clark, 2007, pp. 13-14), que representan un enorme reto - ¿insalvable?- en nuestro contexto sociopolítico y económico actual:

1. La globalización fomenta una nueva cultura política (interés en la inclusión, nuevos modos de gobernanza);

2. El consumo en general y la demanda de recreación aumentan con mayores ingresos y educación, siendo el turismo y la actividad cultural parte de esta transformación;

3. La cultura no se mantiene aislada. Se amplía incesantemente a través de la apertura de nuevos espacios para producir arte y cultura, y actividades artísticas y recreativas que crean escenarios cuyos atributos son identificables por públicos específicos (compradores y consumidores);

4. La ciudad aumenta la capacidad de atraer visitantes cuyo interés es el consumo de la cultura en actividades artísticas y recreativas.

Las industrias de servicios, ¿'son "industrias del futuro"?

Históricamente, el desarrollo tecnológico ha sido un importante motor en la reconfiguración de los sectores económicos (Robertson, 2017). El actual -y futuroavance que ha tenido la tecnología, definido por algunos autores como la "cuarta revolución industrial” (Ford, 2015; Schwab, 2016), no ha sido la excepción. Actualmente, hay evidencia de que la digitalización, robotización y continua mejora de los sistemas computacionales "inteligentes" están generando la transformación de ciertas industrias: por ejemplo, la adopción de nuevas formas de organización de sus cadenas de valor (Mudambi, 2008); la desaparición de ciertas industrias (Brynjolfsson \& McAffe, 2016): por ejemplo, Kodak, empresa de equipamiento fotográfico, declarada en quiebra en 2010 como resultado de los cambios en el 
mercado de la fotografía digital; y el desarrollo de nuevas industrias: por ejemplo, la robótica, la genómica y la industria financiera (a partir del desarrollo del Bitcoin), la computación cuántica y el avance de la inteligencia artificial.

Uno de los resultados de los anteriores cambios es un proceso de reconfiguración del empleo y, en consecuencia, de los sectores económicos (Autor \& Salomons, 2017), que consiste en un doble proceso que ocurre de forma simultánea. Por un lado, un deskilling effect en muchos sectores, sobre todo en aquellos cuyos empleos llevan a cabo actividades repetitivas (middle-skill), vinculados con las manufacturas y las actividades agrícolas; por el otro, un reskilling effect, que consiste en desarrollar y simultáneamente eliminar tipos de conocimiento (Bravo Orellana, 2015, p. 6). El objetivo central de estos cambios es la reducción en los costos de producción, concentrándose recursos para mantener un constante proceso de acumulación de conocimiento y generación de innovación.

Diversos autores han señalado que el anterior proceso, especialmente en países desarrollados, resulta en una pérdida absoluta de empleos y en un aumento de desigualdades tanto en el ingreso como en el empleo (Bourguignon, 2017). El caso es que se produce una progresiva bifurcación de la actividad económica en dos categorías: i) servicios intensivos en conocimiento (sIC), cuyos empleos requieren alto contenido de conocimiento y creatividad con elevados salarios, en los que la generación y adopción de alta tecnología (4.0) es posible; y ii) servicios no intensivos en conocimiento (SNIC), un amplio espectro de empleos que no requieren grandes habilidades o conocimiento, con la posibilidad y riesgo de ser reemplazados por un inminente desarrollo de inteligencia artificial. Sumado a lo anterior, de manera paralela, ocurre una reducción relativa y absoluta de empleos en las manufacturas (middle-skills jobs), como los denominan Florida y Mellander, 2014; Autor, Katz y Kearney, 2006.

Los cambios anteriores también han traído consigo una redefinición funcional de las actividades económicas. En efecto, los sic, en particular, han sido señalados como una pieza clave en el marco actual de una economía basada en la constante producción de conocimiento, innovaciones y elementos simbólicos (Den Hertog, 2000; Galloug \& Savona, 2009; Sheamur, 2012; Siggard, 2012).

La importancia del primer grupo de servicios en algunas economías urbanas, especialmente de ciudades europeas y norteamericanas, radica en: i) su alto contenido de empleos con importantes niveles de conocimiento y creatividad (Florida, 2010; Miles, 2007; Wood, 2002); ii) su función como "puentes" o "articuladores" de conocimiento dentro de los sistemas de producción e innovación (Den Hertog, 2000; Piore \& Sabel, 1984; Simmie, 2003); iii) su capacidad de articularse a las redes de conocimiento que se desenvuelven en distintas escalas espaciales (Simmie \& Strambach, 2006; Strambach, 2008); iv) su capacidad de generar nuevas soluciones (Strambach, 2008); v) su potencial para responder a futuras necesidades de nuestra sociedad global en el ámbito de la producción y el consumo (Daniels \& Bryston, 2002; Hall, 2009); y vi) el soporte que ofrecen para el desarrollo de nuevas empresas (Andersson \& Hellerstedt, 2009).

Así, los sic llevan a cabo funciones esenciales en el desarrollo de conocimiento científico (por ejemplo, genética, nanotecnología, medioambiente), tecnológico 
(robótica, informática, comunicaciones), profesional (finanzas, negocios) y simbólico (diseńo, música, cine, teatro) (Graizbord \& Santiago, 2019). A partir de los anteriores cambios y por sus características, es posible definir los sIC como industrias que forman parte de las llamadas 'industrias del futuro' (Ross, 2016) o aquellas responsables de una importante parte del futuro desarrollo de la nueva economía basada en el conocimiento.

La geografía de los SIC, especialmente de los sic-simbólicos, no es azarosa. La literatura indica que la localización y el crecimiento de los sic-simbólicos ocurre principalmente en las grandes ciudades, resultado de las economías de aglomeración que aprovechan (Mossig, 2011; Sobrino, 2016). Sin embargo, el desarrollo tecnológico ha permitido que otras ciudades de diversos tamaños que reúnen una serie de condiciones locales también sean sedes de industrias simbólicas. Entre tales condicionantes se han descrito: i) altos índices de educación (Ko \& Mok, 2014); ii) particulares estilos de vida (Denis-Jacob, 2012); iii) amplia dotación de amenidades urbanas (Florida, 2005); iv) encadenamiento con otros sectores económicos (intensivos y no intensivos en conocimiento) (Markusen \& Schorck, 2006); y v) atractivos naturales y la proximidad a las grandes ciudades (Martin, 2012; Polèse, 2012). Ya desde Alonso (1971), se considera que tales características no necesariamente responden al criterio convencional del tamańo, especialmente porque la magnitud no es precisamente un fenómeno unidimensional.

En este trabajo nos proponemos responder las siguientes preguntas: i) ¿En qué medida las ciudades del país se especializan en sic-simbólicos y sus subgrupos de actividad?; ii) ¿Pueden las actividades especializadas en sIC-simbólicos constituirse en los "motores" del desarrollo de estas ciudades?; y iii) La presencia y la dinámica de sic-simbólicos en una ciudad ¿están asociadas con los factores señalados por la literatura como determinantes de su localización? La hipótesis que guía el trabajo supone que la localización y el desarrollo de los sic-simbólicos no responden exclusivamente al tamańo de las ciudades, sino a una serie de condiciones económicoespaciales, mencionadas previamente, que les permiten colocalizarse y crecer de manera sobresaliente en ciudades que ocupan niveles inferiores en la jerarquía urbana. De ser así, ello subrayaría la importancia de la proximidad espacial para el desarrollo de este tipo de industrias y sugeriría la configuración de entornos (milieu) que favorecen el intercambio de conocimiento creativo en el ámbito local.

\section{Alcance y metodología}

\section{Definición de SIC-simbólicos y fuentes de información}

Para responder las preguntas del apartado anterior, adoptamos una nueva perspectiva en el estudio del sector servicios a partir de distinguir su actividad económica de acuerdo con su 'intensidad' y 'tipo' de conocimiento (Asheim, 2012; Piketty, 2014; Shearmur \& Doloreux, 2008). Tal distinción permite reconocer su función central en los procesos de generación de innovación (tecnología), descubrimientos (ciencia) y creaciones (artístico-culturales) (Graizbord \& Santiago, 2019). En este estudio nos concentramos exclusivamente en esta última categoría. 
La definición operativa de los sic-simbólicos deriva del trabajo de Santiago (2016, pp. 358-370), donde esos servicios están conformados por 34 subramas (5-dígitos) distribuidas en los sectores 51, 54, 61 y 71 del Sistema de Clasificación Industrial de América del Norte (sCIAN). Debido a la diversidad de casos, se han definido tres grupos de actividad dentro de los sic-simbólicos: i) medios de comunicación masiva, ii) servicios culturales y iii) servicios de diseño (Tabla 1). La variable utilizada para analizar los sIC-simbólicos es el personal ocupado (PO) que aparece en los microdatos de los Censos Económicos de 2004 y 2014 elaborados por el Instituto Nacional de Estadística y Geografía (INEGI), México. ${ }^{2}$

\begin{tabular}{|c|c|}
\hline $\begin{array}{l}\text { ACTIVIDAD POR TIPO DE } \\
\text { CONOCIMIENTO SIC-SIMBóLICOS }\end{array}$ & SUBRAMAS EN SCIAN \\
\hline 1. Medios de comunicación masiva & $\begin{array}{l}51112,51113,51511,51512,51521,51913,54181,54182, \\
54184,54186,54191,54192,61162,71121,71131,71132, \\
71141\end{array}$ \\
\hline 2. Actividades culturales & $\begin{array}{l}51211,51219,51222,51223,51224,61161,71111,71112, \\
71113,71151,71211\end{array}$ \\
\hline 3. Servicios de diseño & $54131,54132,54141,54142,54143,54149$ \\
\hline
\end{tabular}

TABla I | Definición de sic-simbólicos en el scian

FUENTE RECUPERADO DE SANTIAGo (20I6), PP. I3 I-I33

\section{Ámbito espacial de estudio}

Para analizar la distribución espacial de los sic-simbólicos, se incluyen las 384 localidades urbanas (ciudades) que conforman el Sistema Urbano Nacional (sun) de México (Sedesol \& Conapo, 2012). El sun está compuesto por 59 zonas metropolitanas, 78 conurbaciones y 247 centros urbanos mayores de 15.000 habitantes. La importancia de este conjunto de localidades es clara, ya que concentran 81,23 millones de habitantes que representaban 72,31\% de la población del país en 2010 .

\section{Indicadores y modelo estadístico}

Para responder la primera pregunta, se utiliza el cociente de localización (CL), cuya expresión es:

Donde:

$$
C L_{i j}=\left(e_{i j} / e_{j}\right) /(E / E)
$$

$$
\begin{aligned}
& C L=\text { cociente de localización del sector i en la ciudad } j ; \\
& e_{i j}=\text { personal ocupado del sector } \mathrm{i} \text { en la ciudad } \mathrm{j} ; \\
& \mathrm{e}_{\mathrm{j}}=\text { total del personal ocupado en la ciudad } \mathrm{j} ; \\
& \mathrm{E}_{\mathrm{i}}=\text { personal ocupado del sector i en el sun; } \\
& \mathrm{E}=\text { total del personal ocupado en el sun. }
\end{aligned}
$$

El detalle de la información (localidad urbana) utilizada de los Censos Económicos requirió ser procesada directamente en el Laboratorio de Microdatos de inegi en la Ciudad de México a través del proyecto LM-727. Por razones de confidencialidad no se presenta la información de cada ciudad, pero el algoritmo utilizado puede ser proporcionado solicitándolo directamente a los autores. 
Un CL igual a 1,00 significa que la actividad está exactamente representada en la misma proporción del promedio del sun y más de 1,00 indica que la ciudad tiene más que su "parte justa". Cuanto mayor sea el valor del CL, mayor será el grado de concentración del sector i en una ciudad j. Si el CL es menor que la unidad, quiere decir que la actividad esta subrepresentada en esa ciudad.

Para conocer si los sic-simbólicos son "motores" del desarrollo de cada ciudad, analizamos el crecimiento de estos servicios entre 2004 y 2014, por medio del 'incremento porcentual', cuya expresión matemática es:

Donde:

$$
\left.I_{i}=\left(\left(\left(e_{i 1} / e_{i 04}\right)\right)^{(1 / t)}\right)-1\right) \times 100
$$

$\mathrm{Ii}=$ el incremento porcentual del personal ocupado en sic-simbólicos en la ciudad i entre 2004 y 2014;

ei0 4 = el personal ocupado en sIC-simbólicos en la ciudad i en 2004;

ei14 = el personal ocupado en sIC-simbólicos en la ciudad i en 2014; $\mathrm{t}=$ magnitud del periodo de estudio.

Para evaluar si la presencia y el crecimiento de los sic-simbólicos en una localidad están asociados con los factores señalados por la literatura como determinantes, utilizamos el modelo de regresión múltiple siguiente:

$Y_{i}=\beta_{0}+\beta_{1}$ (tamaño de la ciudad) $+\beta_{2}$ (empleo 'intensivo en conocimiento') $+\beta_{3}$ (estructura productiva local) $+\beta_{4}$ (amenidades urbanas) $+\beta_{5}$ (estilo de vida 2$)+\beta_{6}$ (desarrollo local)

Donde:

$\mathrm{Y}=$ toma dos valores: 1) el CL de sic-simbólicos en la ciudad i; y 2) el incremento porcentual (utilizando la fórmula 2) de los sic-simbólicos entre 2004 y 2014 en la ciudad i;

tamaño de ciudad $=\%$ de población en la ciudad i con relación a la población total del sun;

empleo 'intensivo en conocimiento' $=$ CL del PO en SIC (sintéticos: técnicoprofesional, y analíticos: científico) de la ciudad i y el incremento porcentual (utilizando la fórmula 2) de sic (excluyendo simbólicos) en la ciudad i entre 2004 y 2014;

estructura productiva = CL del PO en los sectores: comercio, manufactura, turismo, SNIC;

amenidades urbanas = toma dos formas: amenidades urbanas 1: CL del PO en servicios de esparcimiento; y amenidades urbanas 2: \% de viviendas con servicios de internet (conectividad);

estilos de vida $=\%$ de población con educación superior de la ciudad i; desarrollo local $=$ incremento porcentual (utilizando la fórmula 2) del po en la ciudad i excluyendo 'servicios intensivos en conocimiento' entre 2004 y 2014. 
Las variables 'tamaño de ciudad', 'amenidades urbanas 2' y 'estilo de vida' provienen de 'Principales resultados por localidad (ITER)' del Censo de Población y Vivienda 2010, de INEGI. El 'empleo intensivo en conocimiento', la 'estructura productiva', las 'amenidades urbanas 1' y el 'desarrollo local' fueron construidas con información del po de los Censos Económicos de 2014 obtenidos en el Laboratorio de Microdatos de INEGI con el proyecto LM-727. Las relaciones entre variables se identifican a través de ejercicios de mínimos cuadrados ordinarios (ols), destacando las variables que son significativas para el análisis.

\section{Resultados}

Descriptivos: Importancia de los SIC-simbólicos en el suN

En 2014, el po en México fue de 21,5 millones. Los sic-simbólicos tienen una reducida participación dentro de la economía nacional, pues representaron únicamente $1,38 \%$ del po nacional. El sun concentraba $88,78 \%$ del po nacional. Dentro de la estructura económica del sun, los sic-simbólicos también representaron una reducida proporción, ya que únicamente $1,49 \%$ se empleaba en este tipo de servicios. La estructura productiva de estos servicios en el ámbito urbano del país se distribuye de la siguiente forma: 76,51\% en medios masivos; $15,09 \%$ en arte y cultura; y $8,04 \%$ en diseño. Estas son las proporciones de empleo formal en servicios dedicados a la producción y creación de imágenes, significados, deseos, atributos estéticos, diseños, marcas y símbolos en México y su sistema urbano. Con estos breves datos descriptivos es posible tener una idea de la importancia relativa de los sic-simbólicos en el ámbito urbano de México (Tabla 2).

\begin{tabular}{|c|c|c|c|c|c|}
\hline & \multicolumn{2}{|c|}{ ABSOLUTOS } & \multicolumn{2}{|c|}{$\%$} & \multirow{2}{*}{$\begin{array}{c}\text { INCREMENTO } \\
\text { PORCENTUAL } \\
2004-2014\end{array}$} \\
\hline & 2004 & 2014 & 2004 & 2014 & \\
\hline \multicolumn{6}{|l|}{ México } \\
\hline Total & $16.221,72$ & $21.576,36$ & 100,00 & 100,00 & 2,89 \\
\hline SIC-simbólicos & 230,01 & 296,71 & 1,42 & 1,38 & 2,58 \\
\hline Medios masivos & 154,34 & 224,36 & 0,95 & 1,04 & 3,81 \\
\hline Cultura & 43,34 & 47,79 & 0,27 & 0,22 & 0,98 \\
\hline Diseño & 32,34 & 24,57 & 0,20 & 0,11 & $-2,71$ \\
\hline \multicolumn{6}{|l|}{ SUN } \\
\hline Total & $13.630,35$ & $19.145,36$ & 100,00 & 100,00 & 3,46 \\
\hline SIC-simbólicos & 217,87 & 285,87 & 1,60 & 1,49 & 2,75 \\
\hline Medios masivos & 147,97 & 218,70 & 1,09 & 1,14 & 3,98 \\
\hline Cultura & 38,92 & 43,14 & 0,29 & 0,23 & 1,04 \\
\hline Diseño & 30,98 & 24,02 & 0,23 & 0,13 & $-2,51$ \\
\hline
\end{tabular}

TABLA 2 | México: Descriptivos generales del personal ocupado en SIC-simbólicos, 2004 Y 2014 (MILES)

FUENTE ELABORACIÓN PROPIA CON INFORMACIÓN DE LOS CENSOS ECONÓMICOS DE 2004 Y 2OI4; 'MÉXICO' CON INFORMACIÓN DEL SISTEMA DE AUTOMATIZACIÓN DE INFORMACIÓN CENSAL, SAIC, INEGI (HTTPS://WWW.INEGI.ORG.MX/APP/SAIC/); 'SUN' CON INFORMACIÓN DEL LABORATORIO DE MICRODATOS DE INEGI, PROYECTO DE INVESTIGACIÓN LM-727 
Pregunta 1) ¿En qué medida las ciudades del pais se especializan en SIC-simbólicos y sus subgrupos de actividad? En 2014, únicamente 43 ciudades tenían niveles de especialización sobresalientes $(\mathrm{CL} \geq 1,00)$ de Po en sic-simbólicos como parte de su economía local (Figura 1). Este resultado sugiere la configuración de entornos o milieu que posibilitan el intercambio de conocimiento y la generación de elementos creativos por parte de los sic-simbólicos en el ámbito local. En el resto de las ciudades del sun, los niveles de concentración de los sic-simbólicos indicaban que el empleo en esas actividades no rebasaba "el justo medio" ( $\mathrm{CL}<1,00)$, lo cual no permite considerarlas como importantes polos de atracción de esas y otras actividades complementarias.

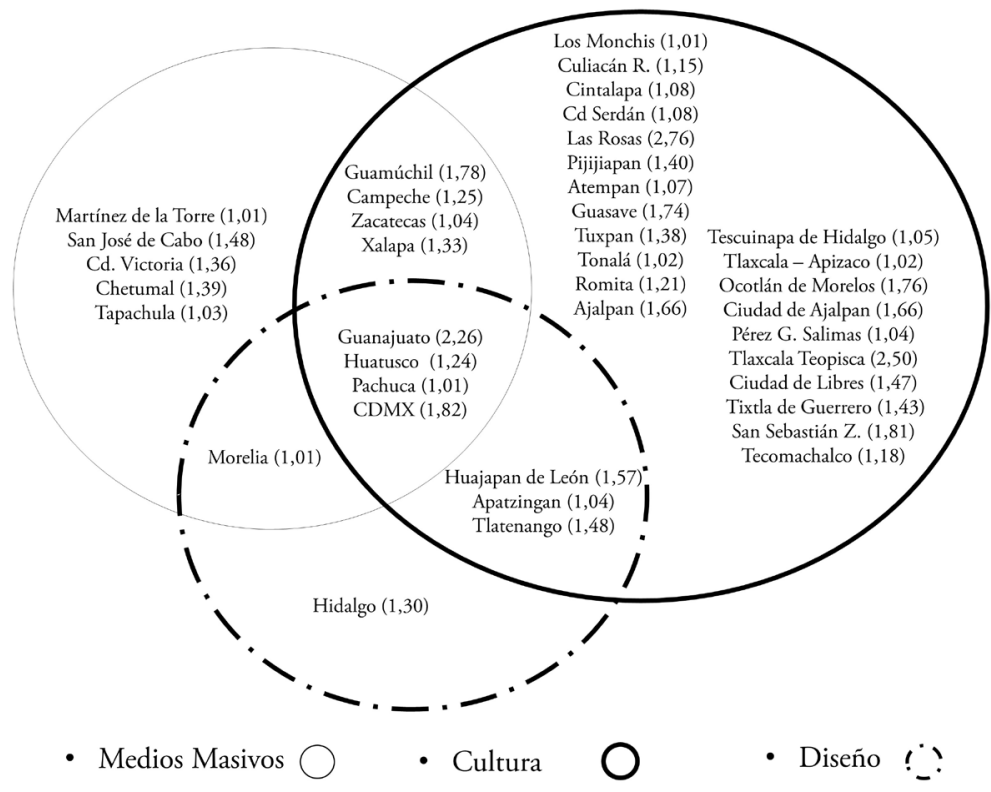

FIgURA I | México: Ciudades especializadas en siC-simbólicos

NOTA EL VALOR ENTRE PARÉNTESIS ES EL COCIENTE DE LOCALIZACIÓN DE SIC-SIMBÓLICOS DE CADA CIUDAD EN $20 I 4$

FUENTE ELABORACIÓN PROPIA CON INFORMACIÓN DEL LABORATORIO DE MICRODATOS DE INEGI, NOVIEMBRE DE 2OI8, PROYECTO LM-727

Las 43 ciudades con sobresalientes concentraciones relativas (o especialización) de sic-simbólicos no tienen el mismo perfil productivo de esos servicios (Figura 1). Cada una se especializa en un subgrupo o bien una combinación particular de actividades según los tres grupos de sic-simbólicos (medios masivos, arte y cultura, y diseño). La Figura 1 ilustra el tipo, la combinación de estas actividades y el valor del CL por ciudad. Hay dos rasgos importantes de destacar.

Primero, los casos de Guanajuato, Ciudad de México, Huatusco y Pachuca, ya que en dichas ciudades se concentran los tres grupos de actividad. La combinación de los tres grupos de sic-simbólicos en la Ciudad de México puede ser explicada por 
ser la capital del país y concentrar la actividad y las instituciones gubernamentales destinadas al desarrollo de las comunicaciones, la cultura y el arte del país (Muñoz \& Gómez, 2011). En Guanajuato, por ser sede del Festival Internacional Cervantino, uno de los principales eventos culturales del país. Estas dos ciudades han sido declaradas por la Unesco como patrimonio de la humanidad por su riqueza arquitectónica. Pachuca, por su localización geográfica, posiblemente está aprovechando su proximidad a la Ciudad de México. Huatusco forma parte de la "ruta del café" en el estado de Veracruz, además de ser próxima a la ciudad de Xalapa, principal centro universitario y cultural de la región. Tales características sugieren que los artistas locales y de otras ciudades del país encuentran en estas ciudades el ámbito para desarrollar sus carreras artísticas, pues además de encontrar los espacios para ello, también se benefician de las instituciones que les dan soporte. Pensamos que la co-localización de los tres grupos de actividad de las anteriores cuatro ciudades abre la posibilidad de que existan ciertas sinergias creativas entre los tres grupos de actividad en la economía local. Para dar seguimiento a esta pista, serían necesarios estudios particulares de corte cualitativo que den cuenta de las redes de intercambio de conocimiento e innovación entre este tipo de servicios.

Segundo, una gran proporción de esas ciudades, sobre todo aquellas especializadas en servicios culturales, son centros urbanos que no ocupan la cúspide del sistema urbano. Se trata de ciudades ubicadas en distintas posiciones de la jerarquía urbana, asociadas a la actividad turística al contar con atractivos naturales (playas, ríos, lagos, reservas naturales), patrimonio arquitectónico (ciudades con arquitectura colonial) y arqueológico, y proximidad a una gran metrópoli.

Pregunta 2) ¿Pueden las actividades especializadas en SIC-simbólicos constituirse en los "motores" del desarrollo de estas ciudades? A partir de la relación entre niveles de concentración relativa (CL) e incremento porcentual (I) de los SIC-simbólicos en el periodo que se analiza, se distinguen cuatro conjuntos o tipos de ciudades.

Tipo I: Ciudades con concentración sobresaliente cuyo crecimiento es mayor que el promedio urbano. Son 29 ciudades que, además de contar con concentraciones por sobre el promedio urbano, tienen un ritmo de crecimiento (I) arriba del promedio y concentraron $61,82 \%$ del crecimiento absoluto de sIC-simbólicos del suN, características que las definen como los principales "motores" de dichas actividades. Tipo II: Ciudades con concentración sobresaliente de estas actividades, pero cuyo crecimiento es menor al promedio urbano. Son 14 ciudades cuya actividad en sIC-simbólicos muestra un ritmo de crecimiento (I) por debajo del promedio y una reducida aportación al crecimiento absoluto de esos servicios (2,36\%) en el sun.

Tipo III: Ciudades con concentración relativa por debajo del "justo medio", pero cuyo crecimiento es superior al promedio urbano. Se trata de 150 ciudades cuya aportación al crecimiento de esos servicios fue de 32,03\%.

Tipo IV: Ciudades que no sobresalen y con un "pobre" crecimiento. Se trata de la mayor parte de las localidades del Sun (189), cuya aportación al total del po en sics-imbólicos en el sun fue de apenas 3,80\%. 


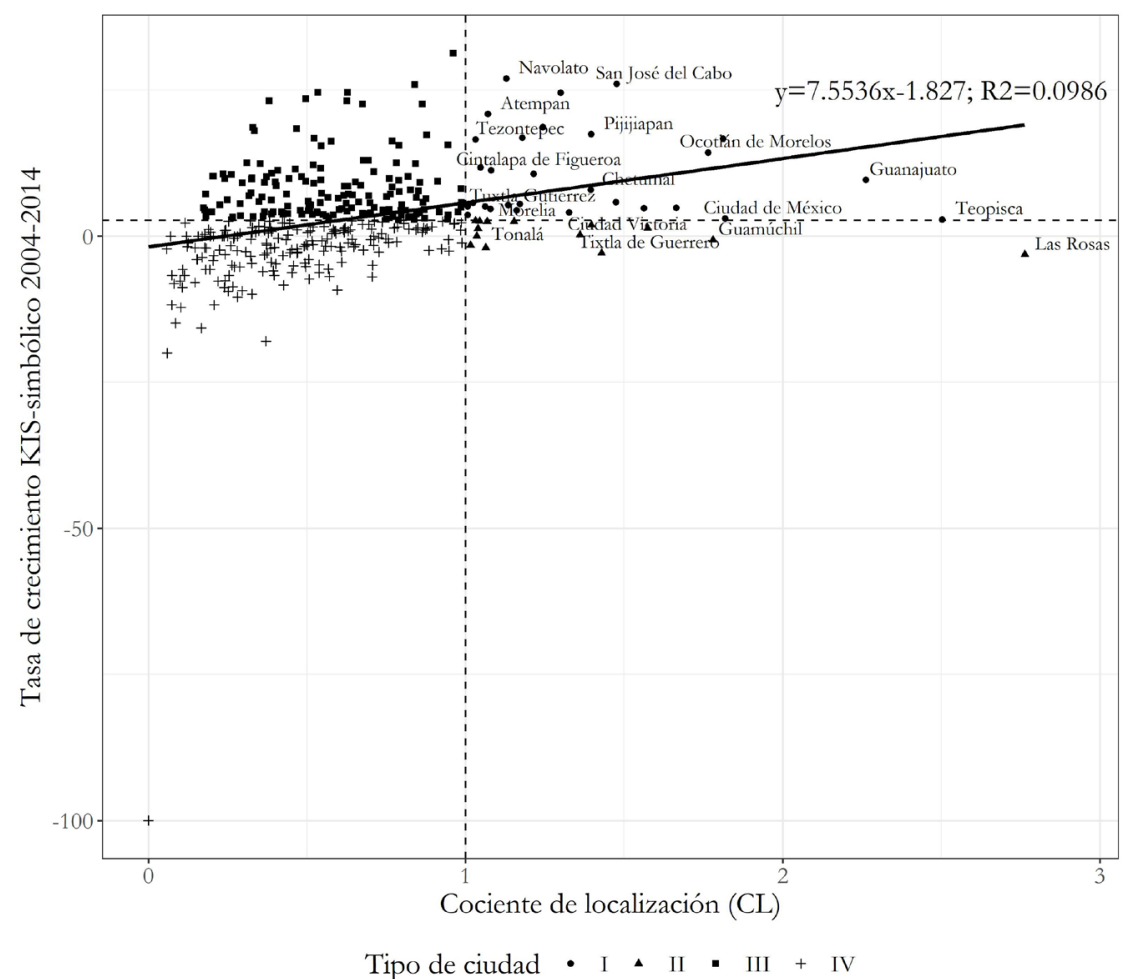

FIGURA 2 | México: Especialización y crecimiento de los SIC-simbólico, 20042014

NOTA LAS VARIACIONES EN LA TASA DE CRECIMIENTO DE LOS SIC-SIMBÓlicos (2004-20I4) SE ENCUENTRAN, AUNQUE DE FORMA REDUCIDA $R^{2}$ AIUSTADA $=9,8 \%$, POSITIVAMENTE ASOCIADAS CON LOS NIVELES DE ESPECIALIZACIÓN EN ESE MISMO TIPO DE SERVICIOS A TRAVÉS DE LAS CIUDADES DEL SUN. PROMEDIO DEL INCREMENTO PORCENTUAL (I) EN EL SUN $=2,74$

FUENTE ELABORACIÓN PROPIA CON INFORMACIÓN DE LOS CENSOS ECONÓMICOS DE 2004 Y 2014 OBTENIDA EN EL LABORATORIO DE MICRODATOS DE INEGI, PROYECTO DE INVESTIGACIÓN LM-727

La anterior dinámica permite definir las ciudades en las que los sic-simbólicos pudieran constituirse en los "motores" del desarrollo de sus economías locales, especialmente aquellas que constituyen el Tipo I. Desde luego, entre este grupo de ciudades se encuentra la Ciudad de México, cuyo tamańo y crecimiento (relativo y absoluto) la definen como el principal núcleo concentrador de estos servicios y es, además, por su diversificación, el centro por antonomasia de estas actividades. La literatura ofrece argumentos para pensar que la tendencia locacional de estos servicios impone una inercia difícil de quebrar por otras ciudades que pudieran atraer este tipo de actividad de sic-simbólicos. Sin embargo, existen ejemplos importantes en donde los SIC-simbólicos resultan un atractivo particular y ofrecen un potencial y una sinergia para el crecimiento económico (véase ciudades Tipo I de la Figura 2). En el siguiente apartado, el análisis se concentra en este grupo de ciudades (Tipo I) para conocer sus características y las variables que están influyendo en su sobresaliente posición en el sistema urbano. 


\begin{tabular}{|l|l|l|l|l|}
\hline \multicolumn{1}{|c|}{ VARIABLES INDEPENDIENTES } & \multicolumn{2}{c|}{ CL $^{\mathrm{A}}$} & \multicolumn{2}{c|}{ I $^{\mathrm{A}}$} \\
\hline Intercepto & 4,018 & $* * *$ & 65,540 & $* * *$ \\
\hline Tamańo ciudad & 6,071 & $* * *$ & $-4,564$ & B*** $^{-1}$ \\
\hline Empleo 'intensivo en conocimiento' & & & & \\
\hline sic-sintéticos (técnico-profesional) & $-0,765$ & $* * *$ & n.a. & - \\
\hline sic-analíticos (científico) & n.a. & - & n.a. & - \\
\hline Crecimiento ${ }^{D}$ sic & 0,052 & $* * *$ & n.a. & - \\
\hline Estructura productiva & & & & \\
\hline Comercio & $-0,724$ & $*$ & $-15,150$ & $*$ \\
\hline Manufactura & $-0,690$ & $* *$ & n.a. & - \\
\hline Turismo (de playa) & n.a. & & n.a. & - \\
\hline sNic & $-1,221$ & $* *$ & 16,730 & $*$ \\
\hline Amenidades urbanas & & & & \\
\hline Tipo 1: Servicios de esparcimiento & n.a. & - & n.a. & - \\
\hline Tipo 2: Viviendas con internet & n.a. & - & 0,000004 & C. \\
\hline Estilo de vida & & & & \\
\hline \% de pob. con educación superior & n.a. & - & n.a. & - \\
\hline Desarrollo local & & & & \\
\hline Crecimiento D del empleo & n.a. & - & n.a. & - \\
\hline R2 ajustada & 0,482 & - & 0,251 & - \\
\hline n & 36 & - & 38 & - \\
\hline p-valor & 0,00002902 & - & 0,004343 & - \\
\hline
\end{tabular}

TABLA 3 | sun: Modelos de regresión de ciudades con especialización ( $\mathrm{CL} \geq \mathrm{I} . \mathrm{OO})$ y su crecimiento (I) de sIC-simbólicos

NOTA SIGNIFICANCIA ESTADÍSTICA: O $*^{* * *}$; O,OOI $=^{* *}$; O,OI $=*$; O,O $5=$; N.A. $=$ NO APORTA AL MODELO. PRUEBAS ESTADÍSTICAS: I) TEST BREUSCH-PAGAN: CON UN NIVEL DE SIGNIFICANCIA DEL 95\%, EXISTE EVIDENCIA PARA NO RECHAZAR LA HIPÓTESIS NULA QUE ASUME HOMOCEDASTICIDAD (VALOR-P $=0,097$ ); II) AUTOCORRELACIÓN DURBIN-WATSON: CON UN NIVEL DE CONFIANZA DEL 95\%, NO SE RECHAZA LA HIPÓTESIS NULA QUE SUGIERE AUSENCIA DE AUTOCORRELACIÓN DE ORDEN UNO (VALOR-P = 0,302); III) MULTICOLINEALIDAD POR FACTOR DE INFLACIÓN VARIANZA (VIF): TODAS LAS VARIABLES REGISTRARON UN VIF MENOR DE IO, POR LO TANTO SE ASUME AUSENCIA DE MULTICOLINEALIDAD EN LOS ERRORES DE LAS VARIABLES INDEPENDIENTES.

a valores de las ciudades del sun con un 'Cociente de localización' (Formula i) MAYOR O IGUAL A I,OO.

B LOgaRitmo DE LA POBlación EN LA CiUdAd i.

C SE CONTROLÓ POR ESTA VARIABLE PARA EVITAR MULTICOLINEALIDAD Y HOMOCEDASTICIDAD EN EL MODELO.

D HACE REFERENCIA AL INCREMENTO PORCENTUAL A PARTIR DE LA FORMULA (2)

FUENTE ELABORACIÓN PROPIA. INFORMACIÓN DEL EMPLEO EN SIC-SIMBÓLICOS PARA 'CL' E 'I' CON INFORMACIÓN DEL LABORATORIO DE MICRODATOS DE INEGI, NOVIEMBRE DE 2OI 8, PROYECTO LM-727

Pregunta 3) ¿Qué factores señalados por la literatura están determinando la localización y la dinámica sobresaliente de los SIC-simbólicos? Las variables señaladas por la literatura que determinarían la localización de los sic-simbólicos a través de un conjunto de ciudades explican casi $50 \%\left(\mathrm{R}^{2}\right.$ ajustada=48,17\%) de las variaciones en el CL de esos mismos servicios en el conjunto de las ciudades Tipo I. Sin embargo, no todas 
esas variables son significativas y su relación con el CL de los SIC-simbólicos no es unidireccional (Tabla 3).

Las variables que influyen de forma positiva en la especialización de los sic-simbólicos son el tamaño de la ciudad y la tasa de crecimiento del empleo en sic. La primera variable, asociada ampliamente con este tipo de servicios (véase Currid \& Scott, 2013; Polése, 2012), hace referencia a por lo menos dos aspectos: a) la amplitud del mercado de clientes y proveedores que requieren estos servicios para sostener su actividad económica, lo cual aumenta su volumen y diversidad conforme se incrementa el tamańo de las ciudades (Jacobs, 1969); y b) las economías de aglomeración que tienen una relación positiva con la posición de las ciudades en la jerarquía urbana. La segunda variable también tiene dos aristas: i) subraya la importancia que tienen los 'sectores intensivos en conocimiento' en la economía local, ya que sus empleados son poseedores de altos ingresos, particulares estilos de vida y características de consumo artístico-cultural por arriba del promedio (Florida, 2005; Florida \& Mellander, 2014); y ii) indica que la sola presencia de sic no es suficiente para generar importantes concentraciones espaciales de los sic-simbólicos, sino que una pieza clave es el ritmo de crecimiento que tienen otros sic en la economía local.

Por otro lado, los niveles de especialización de los sIC-simbólicos son influidos de forma negativa por los niveles de concentración relativa de servicios no intensivos en conocimiento (SNIC), manufactura, comercio y sIC-sintéticos. Este resultado, similar a lo registrado por otros investigadores en países como Canadá o Australia (Middleton \& Freestone, 2008; Waitt \& Gibson, 2009), es explicado por las características propias del empleo de las anteriores actividades, ya que son empleos con habilidades y educación de niveles bajo y medio, que no reciben altos salarios y cuyas posibilidades de consumo son reducidas. Incluso, las investigaciones que han llegado a similares resultados han mostrado que los empleados en las industrias de minería y manufactura carecen de interés en las actividades relacionadas con cuestiones simbólicas y se sienten privados del derecho a ellas, lo cual puede llegar a inhibir el desarrollo de los sic-simbólicos en la economía local. La presencia de los sic-sintéticos (técnico-profesional) en este grupo de variables refuerza el último planteamiento del párrafo anterior, en el que se indica la importancia del crecimiento de los SIC más que su sola presencia en la economía local.

Las variables señaladas previamente tienen un nivel menor de explicación sobre las variaciones en el crecimiento de los sic-simbólicos entre las ciudades de Tipo I $\left(\mathrm{R}^{2}\right.$ ajustada $\left.=0,25\right)$ (Tabla 3). Los resultados indican que únicamente las variables 'tamaño de ciudad', 'comercio' y 'sNic' son relevantes en el análisis, aunque sus efectos tienen diferente sentido. La primera es indicativa de que el principal crecimiento de los sic-simbólicos no está ocurriendo en las grandes ciudades del país, sino en ciudades que ocupan otras posiciones en la jerarquía urbana, tal como fue señalado previamente. La segunda refuerza la idea expuesta en el apartado anterior sobre la relación negativa entre la co-localización de sic-simbólicos y ciertos sectores con bajos niveles adquisitivos y educativos. La tercera sugiere que al incrementar el nivel de presencia de sic-simbólicos en una ciudad tiene lugar un proceso de bifurcación o polarización del empleo del sector servicios local. De acuerdo con Lindley y Machine (2013), la presencia de este último proceso en una ciudad es explicado 
por los patrones de consumo y los niveles de ingresos con los que cuenta el personal ocupado en SIC, y les permite tener una mayor demanda de servicios con medios o bajos niveles de calificación. Este resultado no contradice lo señalado en el párrafo anterior, sino que muestra uno de los efectos del crecimiento de los sic-simbólicos en una ciudad, lo cual no necesariamente significa que la presencia de SNIC tenga un efecto positivo en la concentración relativa de esos servicios. Por el contrario, la relación identificada entre estas dos variables (sIC-simbólicos y SNIC) abre una interesante veta para futuras investigaciones, pues podría ser un signo de un posible efecto negativo del desarrollo de servicios culturales en una ciudad.

Por último, es interesante que, contrario a lo esperado, las variables 'población con educación superior', 'conectividad' (viviendas con internet), y 'amenidades de esparcimiento' no resultaron significativas. Este resultado se debe posiblemente a que dichas variables se encuentran comprendidas en la variable 'tamaño de las ciudades' (Glaeser, 2012).

\section{Reflexiones finales}

En este trabajo se analiza la participación y los cambios de los sic-simbólicos entre las 384 ciudades que constituían el sun de México en 2004 y 2014. Los resultados indican que 43 ciudades tienen sobresalientes concentraciones relativas (o especialización) en alguno o varios de los tres grupos de actividad de los sic-simbólicos: i) medios masivos, ii) diseño y iii) arte y cultura. Este resultado subraya la importancia de la proximidad espacial para el desarrollo de este tipo de industrias y sugiere la configuración de entornos o milieu que posibilitan el intercambio de conocimiento y la generación de elementos creativos por parte de los sic-simbólicos en el ámbito local. Resultan interesantes los casos de las ciudades de Guanajuato, Ciudad de México, Huatusco y Pachuca, donde existe la colocalización de los tres grupos de sic-simbólicos, lo cual abre una doble posibilidad. Por un lado, la existencia de ciertas sinergias creativas entre los tres grupos de actividad en la economía local, lo cual se constituiría como un activo clave para su desarrollo. Pero, por el otro, la posibilidad de que dicha colocalización se trate de una 'mezcla no relacionada', como lo sugieren Asheim y Hansen (2009), que demandaría buscar alternativas para su atención. Conocer cuál de estas posibilidades está ocurriendo requiere futuros estudios -cualitativos- de cada ciudad.

Los niveles de concentración relativa (especialización) y crecimiento (positivo y negativo) de los sic-simbólicos no se encuentran asociados con el peso demográfico de las ciudades del país. En cambio, la mayor parte de las ciudades que cuentan con concentraciones sobresalientes de sic-simbólicos tienen como rasgo común un amplio patrimonio arquitectónico o ser sedes de festivales que representan un nicho que atrae visitantes e ingresos, y que se han convertido en su marca y sello simbólico. Estos resultados abren un abanico de posibilidades para promover las estrategias de la política cultural orientadas a impulsar la localización y el desarrollo de los sic-simbólicos entre las ciudades. Si bien algunas de esas políticas han sido sugeridas de manera general por Sasaki (2008), el ejercicio estadístico permite identificar variables o criterios mínimos que deberían acompañar su desarrollo. Entre tales 
variables se encuentran el incremento en el nivel de ingreso, el aumento del nivel educativo de la población, el impulso de industrias que ocupen individuos con altos niveles de calificación y la mejora estético-funcional de las ciudades. El desarrollo de estas condicionantes puede significar un importante esfuerzo de los gobiernos locales para reconfigurar social, productiva y espacialmente aquellas ciudades que busquen constituirse como lugares centrales en la dinámica global de producción de actividades artístico-culturales.

Las anteriores reflexiones enfatizan el papel que pueden desempeñar los sic-simbólicos como piezas clave en las economías urbanas de México a través de dos elementos centrales: i) reconocer otras formas de conocimiento o innovaciones distintas al desarrollo tecnológico y científico, como posibles motores de su desarrollo económico y social; y ii) subrayar la importancia del contexto local o milieu de cada ciudad para su concentración espacial y aprovechamiento. La clasificación de los SIC-simbólicos, presentada en el apartado metodológico, contribuye a hacer operativas políticas públicas orientadas a convertir ese tipo de servicios en un activo competitivo dentro de sus economías locales. Finalmente, los anteriores elementos abren una agenda de investigación que, además de ampliar el análisis de las variables que influyen en el crecimiento de este tipo de servicios, debe buscar conocer el efecto multiplicador y los encadenamientos productivos de los sic-simbólicos con otros sectores que también pueden constituirse en la "marca" de cada ciudad (por ejemplo, la gastronomía), sobre todo en el marco de países en desarrollo. Realizar lo anterior permitiría ampliar aún más el espectro de acciones necesarias para impulsar el desarrollo urbano y, en especial, la calidad de vida de la población residente en cada una de las ciudades del sistema urbano nacional, así como abrir un abanico de posibles estrategias orientadas a impulsar la localización y el desarrollo de los SIC-simbólicos en las economías urbanas.

\section{Referencias bibliográficas}

Alonso, W. (1971). The economics of urban size. Papers Regional Science Association, 26, 67-83. https://doi.org/10.1007/BF01908693

Amin, A. \& Thrift, N. (2007). Cultural-economy and cities. Progress in Human Geography, 31(2), 143-161. https://doi.org/10.1177/0309132507075361

Andersson, M. \& Hellerstedt, K. (2009). Location attributes and start-ups in knowledgeintensive business services. Industry and Innovation, 16(1), 103-121. https://doi. org/10.1080/13662710902728126

Asheim, B. (2007). Differentiated knowledge bases and varieties of regional innovation systems. Innovation: The European Journal of Social Science Research, 20(3), 223-241. https:// doi.org/10.1080/13511610701722846

Asheim, B. (2012). Innovating: creativity, innovation and the role of cities in the globalizing knowledge economy. En T. Yigitcanlar, K. Metaxiotis \& F. J. Carrillo (Eds.), Building prosperous knowledge cities: Policies, plans and metrics (pp. 3-23). Cheltenham, UK Northampton, MA: Edward Elgar. 
Asheim, B. \& Hansen, H. K. (2009). Knowledge bases, talents, and contexts: On the usefulness of the creative class approach in Sweden. Economic Geography, 85(4), 425-442. https:// doi.org/10.1111/j.1944-8287.2009.01051.x

Autor, D., Katz, L. \& Kearney, M. (2006). Measuring and interpreting trends in economic inequality: The polarization of the us labor market. AEA Papers and Proceedings-American Economic Association, 96(2), 189-94. http://dx.doi. org/10.1257/000282806777212620

Autor, D. \& Salomons, M. (2017). Does productivity growth threaten employment? Documento preparado para ecb Forum on Central Banking, Sintra, Portugal (pp. 1-74). https://pdfs. semanticscholar.org/107b/63fb2e6794dafe06f9cb056f82c1093253d8.pdf

Avent, R. (2016). The wealth of humans. Work and its absence in the Twenty-first Century. Londres: Penguin Books UK.

Bourguignon, F. (2017). La globalización de la desigualdad. México: Fondo de Cultura Económica.

Bravo Orellana, E. R. (2015). Deskilling, up-skilling or reskilling? Effects of automation in information systems context. Twenty-first American conference on information systems. https://pdfs.semanticscholar.org/0d46/4ed08abb89434c063ea5145564598ac46c8b. pdf

Brynjolfsson, E. \& McAffe, A. (2016). The second machine age. Work, progress, and prosperity in a time of brilliant technologies. NY: Norton \& Company.

Burdett, R. \& Philipp, R. (Eds.) (2018). Shaping cities in an urban age. Londres: Phaidon Press.

Camagni, R. (2016). Afterthoughts on urban economic theory and its focus. Journal of Regional Research, (36), 87-105. https://dialnet.unirioja.es/servlet/articulo?codigo $=5819474$

Camagni, R. (2017). The city of business: The functional, the relational-cognitive and the hierarchical-distributive approach. Quality Innovation Prosperity, 21(1), 31-48. http:// dx.doi.org/10.12776/qip.v21i1.818

Clark, T. N. (2007). Making culture into magic: How can it bring tourists and residents. International Review of Public Administration, 12(1), 13-25. https://doi.org/10.1080/ 12294659.2007 .10805088

Cowan, R., David, P. A. \& Foray, D. (2000). The explicit economics of knowledge codification and tacitness. Industrial and Corporate Change, 9(2), 211-253. https://doi.org/10.1093/ icc/9.2.211

Currid, E. \& Connolly, J. (2008). Patterns of knowledge: The geography of advanced services and the case of art and culture. Annals of the Association of American Geographers, 98(2), 414-434. https://doi.org/10.1080/00045600701879458

Currid-Halkett, E. \& Scott, A. J. (2013). The geography of celebrity and glamour: Reflections on economy, culture, and desire in the city. City, Culture and Society, 4(1), 2-11. https://doi.org/10.1016/j.ccs.2013.01.003

Daniels, P. \& Bryson, R. J. (2002). Manufacturing services and servicing manufacturing: Knowledge-based cities and changing forms of production. Urban Studies, 39(5-6), 977-991. https://doi.org/10.1080/00420980220128408

Den Hertog, P. (2000). Knowledge-intensive business services as co-producers of innovation. International Journal of Innovation Management, 4(4), 491-528. https://doi. org/10.1142/S136391960000024X 
Denis-Jacob, J. (2012). Cultural industries in small-sized Canadian cities: Dream or reality? Urban Studies, 49(1), 97-114. https://doi.org/10.1177/0042098011402235

Desrochers, P. (2001). Geographical proximity and the transmission of tacit knowledge. The Review ofAustrianEconomics, 14(1),25-46.https://doi.org/10.1023/A:1007803520748

Florida, R. (2005). Cities and the creative class. Nueva York: Routledge.

Florida, R. (2010). La clase creativa. La transformación de la cultura del trabajo y el ocio en el siglo XXI. Madrid: Paidós.

Florida, R. \& Mellander, C. (2014), The creative class goes global. En C. Mellander, R. Florida, B. T. Asheim \& M. Gertler (Eds.), The creative class goes global (pp. 1-7). Londres: Routledge.

Ford, M. (2015). The rise of robots. Nueva York: Basic Books.

Gallouj, F. \& Savona, M. (2009). Innovation in services: a review of the debate and a research agenda. Journal of Evolutionary Economics, 19(2), 149-172. https://doi.org/10.1007/ s00191-008-0126-4

Glaeser, E. (2012). Triumph of the city. How our greatest invention makes us richer, smarter, greener, healthier, and happier. Nueva York: Penguin.

Graizbord, B. (2007). Megaciudades, globalización y viabilidad urbana. Investigaciones Geográficas, Boletin del Instituto de Geografia, (63), 125-140. https://www.redalyc.org/ articulo.oa?id $=56906309$

Graizbord, B. \& Santiago, L. E. (2019). Ciudades y conocimiento. Clase creativa y servicios de alto contenido de conocimiento en las zonas metropolitanas de México. En J. Sobrino \& V. Ugalde (Eds.), Desarrollo urbano y metropolitano en México (pp. 205256). Ciudad de México: El Colegio de México.

Hales, M., Peterson, E., Mendoza, A. \& Gott, J. (2014). 2014 Global cities index. Global cities, present and future. A. T. Kearney. http://cdn.plataformaurbana.cl/wp-content/ uploads/2014/08/1409601553_global_cities_present_and_future_gci_2014.pdf

Hall, P. (2000). Creative cities and economic development. Urban Studies, 37(4), 639-649. https://doi.org/10.1080/00420980050003946

Hall, P. (2009). Looking backward, looking forward: The city region of the mid-21st century. Regional Studies, 6(43), 803-817. https://doi.org/10.1080/00343400903039673

Jacobs, J. (1969). The economy of cities. Nueva York: Vintage.

Ko, K. W. \& Mok, K. W. P. (2014). Clustering of cultural industries in Chinese cities. Economics of Transition, 22(2), 365-395. doi:10.1111/ecot.12031

Komninos, N., Pallot, M. \& Schaffers, H. (2013). Special Issue on Smart Cities and the Future Internet, Europe. Journal of the Knowledge Economy, 4(2), 113-34. https://doi. org/10.1007/s13132-012-0083-x

López, M. (2009). Del conocimiento tácito y codificado al conocimiento locacionaltraslacional: aplicación a los distritos industriales. Working Paper No 2009/03. Ingenio csic-Universidad Politécnica de Valencia. http://www.ingenio.upv.es/es/delconocimiento-tacito-y-codificado-al-conocimiento-locacional-traslacional-aplicacionlos\#.XGCtnDNKhPZ.

Markusen, A. \& Schrock, G. (2006). The artistic dividend: Urban artistic specialisation and economic development implications. Urban Studies, 43(10), 1661-1686. https://doi. org/10.1080/00420980600888478 
Martin, R. (2012). Measuring knowledge bases in Swedish regions. European Planning Studies, 20(9), 1569-1582. https://doi.org/10.1080/09654313.2012.708022

Martin, R. \& Moodysson, J. (2011). Innovation in symbolic industries: The geography and organization of knowledge sourcing. European Planning Studies, 19(7), 1183-1203. https://doi.org/10.1080/09654313.2011.573131

Middleton, C. \& Freestone, P. (2008). The impact of culture-led regeneration on regional identity in North East England. Documento presentado en Regional Studies Association International Conference, The Dilemmas of integration and competition, Praga, República Checa, 27-29 de mayo 2008. http://citeseerx.ist.psu.edu/viewdoc/ download?doi=10.1.1.224.5737\&rep=rep1\&type=pdf

Miles, I. (2007). Knowledge-intensive services and innovation. En J. R. Bryson \& P. W. Daniels (Eds.), The handbook of services industries (pp. 277-294). Cheltenham, uK | Northampton, MA: Edward Elgar.

Mossig, I. (2011). Regional employment growth in the cultural and creative industries in Germany 2003-2008. European Planning Studies, 19(6), 967-990. https://doi.org/10. $1080 / 09654313.2011 .568807$

Mudambi, R. (2008). Location, control and innovation in knowledge-intensive industries. Journal of Economic Geography, 8(5), 699-725. https://doi.org/10.1093/jeg/lbn024

Muñoz, L. A. \& Gómez, G. R. (2011). Analysis of the film production district in Mexico City. International Journal of Communications, 5, 844-874.

Piketty, T. (2014). El capital en el siglo XXI. Madrid: Fondo de Cultura Económica.

Piore, M. J. \& Sabel, C. F. (1984). The second industrial divide: Possibilities for prosperity. Nueva York: Basic Books.

Polèse, M. (2012). The arts and local economic development: Can a strong art presence uplift local economies? A study of 135 Canadian cities. Urban Studies, 49(8), 1911-1835. https://doi.org/10.1177/0042098011422574

Robertson, P. L. (2017). Technological diffusion in local, regional, national a transnational setting. En B. Warf (Ed.), Handbook on geographies of technology (pp. 17-35). Cheltenham, uK | Northampton, MA: Edward Elgar.

Rocks, C. (2017). London's creative industries - 2017 update. London Datastore. https://data. london.gov.uk/blog/londons-creative-industries-2017-update/

Rodrik, D. (2016). Premature deindustrialization. Journal of Economic Growth, 21(1), 1-33. https://doi.org/10.1007/s10887-015-9122-3

Roman, M. \& Moodysson, J. (2011). Innovation in Symbolic Industries: The Geography and Organization of Knowledge Sourcing. European Planning Studies, 19(7), 1183-1203. https://doi.org/10.1080/09654313.2011.573131

Rosenthal, S. \& Strange, W. (2001). The determinants of agglomeration. Journal of Urban Economics 50(2), 191-229. https://doi.org/10.1006/juec.2001.2230

Ross, A. (2017). The industries of the future. Nueva York: Simon \& Schuster.

Santiago, L. E. (2016). Ciudades, conocimiento e innovación: Estructura y distribución espacial de los servicios intensivos en conocimiento entre las zonas metropolitanas de México. (Tesis doctoral inédita). Ciudad de México: El Colegio de México. https://colmex.userservices. exlibrisgroup.com/view/delivery/52COLMEX_INST/1270171390002716

Sasaki, M. (2008). Developing creative cities through networking. 政策科学 [Journal of Public Policy Research], 77-88. http://r-cube.ritsumei.ac.jp/repo/repository/rcube/4158/ ps15_3_06sasaki.pdf 
Schwab, K. (2017). La cuarta revolución. Ciudad de México: Debate.

Scott, A. (2006). Creative cities: Conceptual issues and policy questions. Journal of Urban Affairs, 28(1), 1-17. https://doi.org/10.1111/j.0735-2166.2006.00256.x

Scott, A. (2014). Beyond the creative city: Cognitive-cultural capitalism and the New Urbanism. Regional Studies. 48(4), 565-578. https://doi.org/10.1080/00343404.2014.891010

Sedesol [Secretaría de Desarrollo Social] \& Conapo [Consejo Nacional de Población], México. (2012). Catálogo. Sistema Urbano Nacional, 2012. México DF: Secretaría de Desarrollo Social. http://www.conapo.gob.mx/en/CONAPO/Catalogo_Sistema_Urbano_Nacio nal_2012

Shearmur, R. (2012). Are cities the font of innovation? A critical review of the literature on cities and innovation. Cities, 29(2), S9-S18. https://doi.org/10.1016/j.cities.2012.06.008

Shearmur, R. \& Doloreux, D. (2008). Urban hierarchy or local buzz? High-order producer services and (or) knowledge-intensive business services innovation. The Professional Geographer, 60(3), 333-355. https://doi.org/10.1080/00330120801985661

Siggaard, J. H. (2012). The changing role of knowledge economy: Concepts of knowledge and knowledge management. En K. I. Westeren (Ed.), Foundations of the knowledge economy: Innovation, learning and clusters (pp. 15-33). Cheltenham, uk | Northampton, MA: Edward Elgar.

Simmie, J. (2003). Innovation and urban regions as national and international nodes for the transfer and sharing of knowledge. Regional Studies, 37(6-7), 607-620. https://doi. org/10.1080/0034340032000108714

Simmie, J. \& Strambach, S. (2006). The contribution of KIBS to innovation in cities: An evolutionary and institutional perspective. Journal of Knowledge Management, 10(5), 26-40. https://www.researchgate.net/deref/http\%3A\%2F\%2Fdx.doi.org\%2F10.110 $8 \% 2 \mathrm{~F} 13673270610691152$

Sobrino, J. (2016). Entre mitos y realidades: Ciudades mexicanas que concentran clase creativa. Estudios Demográficos y Urbanos, 31(2), 501-522. https://www.redalyc.org/articulo. oa?id=31245858007

Storper, M. \& Scott, A. (2009). Rethinking human capital, creativity and urban growth. Journal of Economic Geography, 9(2), 147-167. https://doi.org/10.1093/jeg/lbn052

Strambach, S. (2008). Knowledge-Intensive Business Services (ківs) as drivers of multilevel knowledge dynamics. International Journal Services Technology and Management. 10(2/3/4), 152-174. https://doi.org/10.1504/IJSTM.2008.022117

Taylor, P. (2014). Extraordinary cities. Millennia of moral syndromes, world systems and city/state relations. Cheltenham, UK | Northampton, MA: Edward Elgar.

Townsend, A. (2013). Smart Cities: Big Data, Civic Hackers, and the Quest for a New Utopia. NY: Norton \& Company.

Waitt, G. \& Gibson, C. (2009). Creative small cities: Rethinking the creative economy in place. Urban Studies, $46(5$ \& 6), 1223-1246. https://doi.org/10.1177/0042098009103862

Wood, P. (2002). Knowledge-intensive services and urban innovativeness. Urban Studies, 39(56), 993-1002. https://doi.org/10.1080/00420980220128417

Yigitcanlar, T., Metaxiotis, K., \& Carrillo F. J. (Eds.) (2012). Building Prosperous Knowledge Cities. Policies, Plans and Metrics. Cheltenham, uK | Northampton, MA: Edward Elgar. 